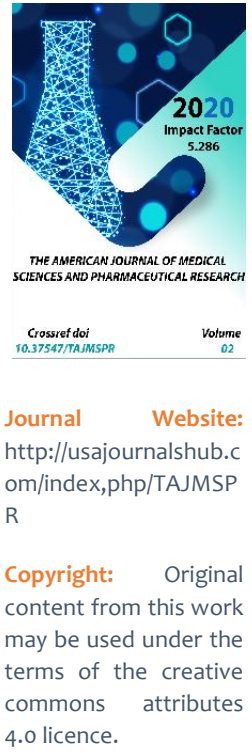

\title{
Endocrine Structures Of The Lungs In Ontogenesis And In Children With Pneumonia
}

Blinova Sofya Anatolyevna

Doctor Of Medical Sciences, Professor Of The Department Of Histology, Cytology And Embryology Of Samarkand State Medical Institute, Uzbekistan

Khamidov Farida Muinova

Head Of The Department Of Pathological Anatomy Of Samarkand State Medical Institute, Candidate Of Medical Sciences, Associate Professor, Uzbekistan

Urakov Kuvandik Nematovich

Assistant Department Of Pathological Anatomy Samarkand State Medical Institute, Uzbekistan

\section{ABSTRACT}

During embryonic histogenesis of human lungs, early morpho- functional differentiation of apudocytes is observed. In the lungs of young children with an inflammatory pathology of this organ, endocrine structures are detected with great constancy, and their number is large. The longer the disease lasts, the more apudocytes and NETs are found in the bronchi and respiratory tract. In large bronchi there are more endocrine structures than in small ones. Inflammatory lung diseases in early postnatal ontogenesis are a predisposing factor for apudocyte hyperplasia.

\section{KEYWORDS}

Apudocytes, respiratory system, endocrinocyte,hyperplasia.

\section{INTRODUCTION}

Endocrine cells of the lungs are often the source of the development of malignant neoplasms. Until now, there is no consensus on the morpho functional features of the 
endocrine cells of the APUD- respiratory system, which are the source of these neoplasms.

Purpose: to determine the dynamics of changes in the endocrine cells of the APUDsystem of the lungs in the fetal period of development, in normal children, as well as in inflammatory pathology of this organ.

\section{MATERIALS AND METHODS}

The material for the study was the lungs of human fetuses (15 cases), children with extra pulmonary pathology (8 cases), and children with acute and chronic pneumonia (12 cases). Paraffin sections were stained by general histological methods, and also impregnated according to the Grimelius method. Applied as electron microscope and morphometric study.

\section{RESULTS AND DISCUSSION}

The lungs of a human fetus at 9-13 weeks of development are at the glandular stage of histogenesis, during which the airways develop. In the lungs of fetuses of 9-10 weeks of development, we did not find endocrine cells in the epithelium of the bronchi and in the epithelial tubes. In the lungs of 11-13-weekold fetuses, apudocytes are located between the cells of the epithelial lining. Many apudocytes are vertically oriented. The processes of endocrine cells in the large bronchi reach the surface of the epithelium, while this is not observed in the smaller bronchi. Lung apudocytes in a fetus of 11 weeks have a well-developed ultrastructure, in comparison with neighboring epithelial cells. Theycontain mitochondria, short cisterns of the granular endoplasmic reticulum and endocrine granules. The cage contains from 8 to 26 granules. They are usually located in the basal part of the cells. In the lungs of an 11week-old fetus, P1 endocrine cells with two types of granules are most often found. In addition to single apudocytes, groups of endocrine cells of three types are also found in the bronchial epithelium of large bronchi. The cells are located at some distance from each other and are interconnected by processes, forming a syncytial structure. In the glandular stage of lung histogenesis, most of the endocrine structures are located in the segmental and interlobular bronchi.

At 15 weeks of embryogenesis, the canalicular stage of lung histogenesis begins, when respiratory bronchioles are formed. The number of apudocytes increases distally. Apudocytes are more common in branched epithelial tubes, which are the rudiments of respiratory bronchioles, than in unbranched ones. They apudocytes usually oval or triangular shaped lumen tubes they do not reach, refer tothe closed type. In the canalicular and alveolar stages of histogenesis, in addition to single endocrinocytes, their accumulations are determined - neuroepithelial bodies (NET).

Moreover, in the canalicular stage increase in the number apudocytes and NET largely occurs in large and medium-sized bronchi and alveolar - apudocytes and NET more consistently identified in terminal bronchioles. With the onset of the alveolar stage, the number of apudocytes increases, while NET, on the contrary, decreases. At 21-22 weeks of intrauterine development, the differentiation of the bronchi and the formation of the respiratory section continue. The number of apudocytes and NET continues to increase by this time. In the bronchial epithelium, along with open-type apudocytes, there is a large number of closed-type endocrine cells. They 
are spread out on the basement membrane and their lateral processes spread far along it.

The branching of the bronchial tree is ahead of the development of the APUD-system endocrine apparatus in it. However, differentiation of apudocytes and NET occurs much earlier than epithelial cells. This indicates their early specific functional activity in embryogenesis. In the proximal parts of the bronchial tree of human fruits, cells of the open type are usually located, while in the distal parts they are of the closed type. In human ontogenesis, the appearance of some types of apudocytes and the disappearance of others occurs in the lungs. Thus, in the glandular stage of histogenesis, we found apudocytes of the $\mathrm{P}_{1}, \mathrm{P}_{2}$, and $\mathrm{P}_{3}$ types, in the canalicular stage - $\mathrm{P}_{2}$ and $\mathrm{P}_{3}$, in the alveolar stage and in newborns - P2 and EC cells. In addition, apudocytes containing granules of type D were found in human lungs. In early ontogenesis in the lungs of human fetuses, "dark" endocrine cells are more common than "light" ones.

In the lungs of neonates number of audiiton much less than in embryos.Most of the bronchi contain 1-2 apudocytes, NET are very rare. The largest number of apudocytes is located in the subsegmental and interlobular bronchi. The total number of endocrine structures in them is, respectively, 4.1 and 2.3 cells per $1 \mathrm{~mm}$ of bronchial length. In the respiratory section, apudocytes and NETs were not found. In children aged 1-4 years, the number of endocrine structures is small.

Theyarefoundonlyinsomesegmental, subsegmental and interlobular bronchi. NET in the lungs in children are rare.Apudocytes and NET are found in all children with inflammatory lung diseases. In a 7-day-old child, in almost every section of the bronchus, there are 1-2 apudocytes and, somewhat less often, NET. Moreover, in the proximal parts of the bronchial tree there are more endocrine structures than in the distal ones. In the respiratory department, NETs are more often determined than apudocytes. A large number of endocrine structures are found in the lungs of a 10-day-old child with pneumonia. A similar picture was also recorded in the lungs of patients with acute pneumonia at the age of 22 and 46 days. They have 5-6 apudocytes per cross section of lobar bronchi, segmental - 2-3 and smaller branches - 0.5-1 apudocytes, NET are determined somewhat less often, they consist of 2-6 cells. In almost every section of the bronchus, there are apudocytes and, less often, NET, consisting of 2-6 cells. In the respiratory section, apudocytes and NET are often located at the site of the transition of bronchioles to the alveolar passages. In these areas, the number of cells in the NET varies widely. In 2- and 4-month-old children with pneumonia, the cross section of the lobar bronchi has 1-2 apudocytes, segmental andsubsegmental bronchi - 5-6, interlobular and intralobular - 2-4, terminal - 0.51apudocytes. Endocrine structures in the lungs of sick children are not only characterized by a large number, but also by their length due to processes spreading along the basement membrane.

The described tendency to an increase in endocrine elements in large bronchi persists in children 6-7 months old with pneumonia. The number of apudocytes in the lobar bronchi increases to $4-6$, in the segmental and especially in the subsegmental - up to 11. The number of NETs is 4-5 and 0.5-1, respectively, per transverse section of the bronchus. In smaller bronchi, the number of apudocytes and NET is approximately within the same 
range as in the previously described periods. At the same time, they were not found in the terminal bronchioles and in the respiratory section. This pattern persists in children who died of pneumonia at an older age. In children aged from birth to 1 year, as well as from 7 months, most apudocytes are detected in large bronchi.Their number per transverse section in the lobar bronchi is 2-3, segmental 2-9, subsegmental and interlobular -4-5, intralobular - 1-3. Only sometimes apudocytes and NETs are detected in the terminal bronchioles, usually no more than 1-2, and also rarely in the respiratory section.

Thus, a single early-specific th-functionally active s-apudocyte is detected in embryogenesis. Most researchers explain their influence on the processes of lung development [ 3 ].In the lungs of young children with an inflammatory pathology of this organ, endocrine structures are detected with great constancy, and their number is large. The longer the disease lasts, the more apudocytes and NETs are found in the bronchi and respiratory tract. In large bronchi there are more endocrine structures than in small ones. Often, hyperplasia of apudocytes in children is accompanied by a number of clinical manifestations [1]. Hyperplasia of apudocytes in the lungs has tumor potential $[4,5]$. Inflammatory lung diseases in early postnatal ontogenesis are a predisposing factor for apudocyte hyperplasia.

\section{CONCLUSIONS}

1. At each stage of histogenesis of the lungs, the endocrine apparatus of the organ has morphological and functional organizational features.

2. In inflammatory diseases of the lungs in newborns and young children, there is a high content of apudocytes and NET.

\section{REFERENCES}

1. Young LR, Brody AS, Inge TH, Acton JD, Bokulic RE, Langston C, Deutsch $\mathrm{GH}$. Neuroendocrine cell distribution and frequency distinguish neuroendocrine cell hyperplasia of infancy from other pulmonary disorders. Chest. 2011 May; 139 (5): 1060-71.

2. Koo CW, Baliff JP, Torigian DA, Litzky LA, Gefter WB, Akers SR Spectrum of pulmonary neuroendocrine cell proliferation: diffuse idiopathic pulmonary neuroendocrine cell hyperplasia, tumorlet, and carcinoids. Am J Roentgenol. 2010 Sep; 195 (3): 661-8.

3. Linnoila RI. Functional facets of the pulmonary neuroendocrine system Laboratory Investigation (2006) 86, 4254440

4. Rizvi SM, Goodwill J, Lim E, Yap YK, Wells AU, Hansell DM, Davis P, Selim AG, Goldstraw $P$, Nicholson AG. The frequency of neuroendocrine cell hyperplasia in patients with pulmonary neuroendocrine tumors and nonneuroendocrine cell carcinomas. Histopathology.2009 Sep; 55(3): 332-7.

5. DaviesSJ, Gosney R, HansellDM, WellsAU, du BoisRM, BurkeMM, MN Sheppard, and NicholsonAG. Diffuse idiopathic pulmonary neuroendocrine cell hyperplasia: an under-recognized spectrum of disease. Thorax. Mar 2007; 62 (3): 248-252. 